\title{
En tiempos de pandemia: de la educación presencial al entorno virtual y de regreso
}

\author{
Claudia Patricia Contreras \\ ccontreras@uach.mx \\ Universidad Autónoma de Chihuahua \\ Chihuahua, México \\ María Teresa Pérez Piñón \\ mperez@uach.mx \\ Universidad Autónoma de Chihuahua \\ Chihuahua, México \\ David Picazo \\ ipicazo@uach.mx \\ Universidad Autónoma de Chihuahua \\ Chihuahua, México \\ Dagoberto Pérez Piñón \\ dperezp@uach.mx \\ Universidad Autónoma de Chihuahua \\ Chihuahua, México
}

\section{RESUMEN}

Durante el 2020 y el 2021 los educadores nos enfrentamos a un panorama de incertidumbre y enorme reto educativo al tener que dar continuidad a los programas de estudio a distancia, evidenciando la falta de preparación ante un acontecimiento de esta naturaleza en el ámbito de la educación superior en México y en el mundo. El presente trabajo es una reflexión sobre la importancia de contar con un plan para continuar las clases ante los retos a los que los docentes y alumnos se enfrentan a partir del distanciamiento social ocasionado por el Covid-19.

Palabras clave: covid-19; pandemia; educación a distancia; educación presencial 


\title{
In pandemic times: from face-to-face education to the virtual environment and back again
}

\begin{abstract}
In 2020 and 2021, educators faced a panorama of uncertainty and an enormous educational challenge due following up distance learning programs. This crisis evidenced the lack of preparation for an event of this nature in the field of higher education in Mexico and the world. This paper is a reflection on the importance of having a plan to continue classes in the face of the challenges encountered by teachers and students as a result of the social distancing caused by Covid-19.
\end{abstract}

Keywords: covid-19; pandemic; distance education; face-to-face education

Artículo recibido: 30 noviembre. 2021 Aceptado para publicación: 29 diciembre 2021 Correspondencia: mperez@uach.mx Conflictos de Interés: Ninguna que declarar 


\section{INTRODUCCIÓN}

La presencia de un nuevo evento patológico, manifestado en el organismo humano, supone de manera tajante una situación compleja, principalmente si el microorganismo se transmite de manera vertiginosa y en el momento no se cuenta con los medios y medicamentos para ser tratado (Trilla, 2020). Tal es el caso de la presencia del virus Sarscov 2, mismo que dio origen a la enfermedad llamada Covid-19, y que el 11 de marzo del 2020 fue declarada como pandemia por la Organización Mundial de la Salud (OMS).

El número de casos por esta patología aumentó de manera acelerada a nivel global, situación que alarmó a la OMS y a los gobernantes de cada país por la rápida propagación del virus y la pasividad en las acciones a tomar para la contención de la enfermedad (Cucinotta \& Vanelli, 2020). La pandemia obligó a las diferentes instituciones educativas de todos los niveles a modificar sus modelos de impartición de clases, hasta ese momento presenciales, y que abruptamente cambió a entornos virtuales (González Fernández, 2021). En el proceso, se vieron afectados aproximadamente mil millones de estudiantes de todos los niveles educativos, de 143 países afectados por la pandemia (Unesco, 2021). En México, el 23 de marzo de 2020, la Secretaría de Educación Pública (SEP) estableció que todas las escuelas prescindieran de sus actividades presenciales. El desafío del sistema educativo fue mayúsculo, la situación de pandemia provocó incertidumbre, dudas e inseguridades entre los actores educativos de nuestro país. Las escuelas cerraron sus puertas físicas y, a pesar de las condiciones, todas las instituciones educativas, de todos los niveles, decidieron adoptar la tele-educación. En este contexto, tanto estudiantes como profesores, administradores escolares y padres de familia se vieron en la imperiosa necesidad de aprender a manejar los entornos virtuales (Muñiz \& Corduneanu, 2020) y de adaptarse a las nuevas condiciones académicas, vinculadas con preservar la salud individual y comunitaria (Cervantes-González \& Hernández López, 2020).

En la decisión de migrar con urgencia de clases presenciales a virtuales, fue notoria la existencia de una brecha en el manejo de las competencias digitales y metodología docentes; la evolución en este sentido se dio de manera acelerada, aunque la academia se impartió sin planificación, ni capacitación de los actores involucrados (García-Peñalvo \& Corell, 2020). En esta contingencia académica, no solo se experimentaron cuestiones negativas, Sierra Pérez (2005), mencionado por Ochoa Gutiérrez \& Balderas Gutiérrez (2021), refiere que se desarrolló el aprendizaje autónomo y autogestivo. Tanto estudiantes 
como docentes y padres de familia se vieron obligados a crear sus métodos de estudio y gestionar su conocimiento a través de la búsqueda en diferentes plataformas, entre otros medios. Sin duda, la pandemia marcó una evolución en el sistema de enseñanzaaprendizaje; los propios docentes debieron aprender a aprender y a desaprender, así como aplicar habilidades y estrategias que los acreditaran como formadores competentes en un contexto retador y desfavorable.

En el contexto actual, surge un nuevo concepto de educación: la educación híbrida. Guaman Chavez et al. (2020) mencionan que la mayoría de las escuelas de los diferentes niveles educativos se han visto en la necesidad de adoptar este modelo de aprendizaje; que es la combinación de la educación tradicional-presencial con la digital-remota, en la que se aprovecha la independencia del estudiante-familia para autogestionar su propio conocimiento.

Por su parte, Méndez Landa (2021) reflexiona sobre las dificultades que aparecieron en el camino, como las distracciones en el hogar, la incapacidad para manejar la tecnología y las fallas de conectividad a Internet. Ante estas dificultades, el autor sugiere que en realidad el tiempo dedicado a la docencia ha sido escaso, y que ha habido una gran pérdida de tiempo para el desarrollo de la clase.

Hoy por hoy, a casi dos años de que se cerraron las puertas físicas de los planteles educativos en México, las Secretarías de Educación y de Salud toman la decisión de abrirlas nuevamente. Hugo López-Gatell, Subsecretario de Salud, aseguró que el regreso a clases presenciales no conlleva riesgo de contagio de Covid-19, por lo que, independientemente del color del semáforo epidémico, las escuelas reabrirán, siempre y cuando se respeten las medidas de contención. Además, se ha dado la opción de que, si algunos integrantes de la sociedad no desean regresar a clases presenciales-tradicionales, las tomen de manera virtual (De la Rosa, 2020). El retorno a clases presencialestradicionales es inminente; el reto al que se enfrentaron profesores, estudiantes y padres de familia durante la pandemia fue colosal para muchos, para otros fue un poco más digerible, sin embargo, complicado, puesto que recurrir al uso de las tecnologías de la información y comunicación (TIC) sin una preparación o capacitación adecuada constituyó un verdadero problema. A lo anterior es necesario sumar la planeación improvisada de los cursos y el obstáculo por el desconocimiento de las plataformas educativas (Cabrera Pérez, 2020). 


\section{DESARROLLO}

El regreso a clases representa un proceso complejo. Farías Soares \& Schoen (2020) llevaron a cabo una revisión de los protocolos para la reapertura de escuelas, publicado hasta el 10 de julio de 2020, en donde se analizaron documentos de 13 países. Destacan que:

"Los países europeos han seguido una ruta propuesta por la Unión Europea para considerar la reapertura de las escuelas, que implica tres criterios: (1) epidemiológico, es decir, que la propagación del virus se haya reducido; (2) un sistema sanitario suficientemente equipado para atender los casos graves, especialmente los que requieren hospitalización; y (3) el seguimiento o la capacidad de realizar pruebas de detección a gran escala para identificar brotes" (p. 5).

La planificación detallada y las medidas preventivas para frenar el Covid-19 son necesarias para poder migrar hacia la educación presencial. Es por ello que, con el fin de proporcionar datos que ayuden a las autoridades a tomar las decisiones correctas para el regreso a clases presenciales en México, Rubio Álvarez et al. (2020) llevaron a cabo una revisión sistemática de las modalidades y estrategias para el regreso a las aulas y la educación híbrida alrededor del mundo. Los resultados indican que la tendencia para retornar a las escuelas es mantener los protocolos de seguridad y sistemas híbridos de educación que la OMS y los gobiernos recomendaron para enfrentar la contingencia. En Latinoamérica, Uruguay es el primer país de la región que proyecta la reapertura de su sistema educativo. Brasil, a pesar de ser el segundo país del mundo con mayor número de casos de Covid-19, planea la reapertura de las escuelas privadas. De la misma manera, Colombia y Perú reabrieron las escuelas en agosto y septiembre de 2021 (pp. 5-6).

La reactivación de la educación en Europa ha sido gradual y de una forma más conservadora, permitiendo que los estudiantes asistan en pequeños grupos, además de mantener la educación superior en forma virtual. Este es el caso de España e Italia, en donde los estudiantes regresaron a clases presenciales en septiembre de 2021 a pesar de que han sido de los países más afectados por la pandemia.

Por su parte, Alemania y Francia consideran un regreso a través del modelo híbrido, sin un distanciamiento social obligatorio, pero con medidas sanitarias. En Suecia no se cerraron las escuelas, sino que se implementaron medidas de distanciamiento social, 
admitiendo un máximo de 15 alumnos por clase, mientras que en Inglaterra se pospuso el regreso hasta septiembre de 2021 debido a nuevos contagios. En Asia, China, Japón y Corea del Sur han tomado medidas más estrictas para sus escuelas primarias y secundarias, manteniendo el distanciamiento social, la alternancia y sin la posibilidad de conversación entre compañeros (Simon, 2020).

En Corea y en China se han llevado a cabo cuarentenas estrictas, pero, aun así, las familias sí quieren el regreso a clases, pues en estos países la presión escolar es muy alta y se quiere volver a la normalidad lo antes posible. Los estudiantes de educación básica y medio superior regresaron a clases, pero las universidades se mantienen cerradas funcionando de manera virtual. En Japón se cerraron las escuelas, ya que en la ciudad de Kitakyushun, se registraron 119 casos en solo 11 días, y lo mismo sucedió en Beijing, China (Taylor, 2020). En Estados Unidos las escuelas regresaron en septiembre de 2021 y, gracias a la implementación de pruebas de Covid-19 en los campus, se ha encontrado que existe una transmisión limitada. Sin embargo, falta un sistema nacional de seguimiento de las infecciones que permita saber cuántos estudiantes se han visto afectados por contagios en la escuela, de manera que se han visto en la necesidad de enviar a miles de niños a casa en los estados con baja tasa de vacunación (Goldstein, 2021).

En la página oficial del Departamento de Educación de los Estados Unidos (U.S. Department of Education, 2021) se pone a disposición de los ciudadanos el Plan de Rescate Estadounidense a través del Mapa de Regreso a Clases. Este plan se enfoca en el regreso a clases, para lo cual, el gobierno invirtió 122 mil millones de dólares a través del Fondo de Ayuda de Emergencia para Escuelas Primarias y Secundarias para apoyar en la prevención del virus. Con estos fondos se pretende mantener a los estudiantes seguros al invertir en orientación para la prevención de enfermedades, equipo de protección y mejorar la ventilación en las aulas, así como la contratación de enfermeras y personal de limpieza para mantener los lugares más seguros. El plan también contempla apoyo para la salud mental.

En México, las Secretarías de Educación y de Salud decidieron el protocolo de inoculación con la vacuna CanSino para los maestros de las escuelas públicas y privadas durante los meses de abril y mayo de 2021 (Núñez et al., 2021). 
"La SEP actualizó la Guía de orientación para la reapertura de las escuelas ante COVID-19, en la que se establece que la reapertura de las escuelas dependerá de la evolución de los escenarios epidemiológicos en cada entidad (SEP, 2021). Recordando que, en el color verde es cuando las escuelas pueden abrir, y en el amarillo solo se aplica la estrategia de Centros de Aprendizaje Comunitario (CCA)" (p. 21).

En México, el 7 de junio de 2021 se autorizó el regreso a clases voluntario, sin embargo, estados como Baja California Sur, Guerrero, Hidalgo, Oaxaca, Puebla, Querétaro, Quintana Roo, Sonora, Tabasco, Tlaxcala y Yucatán optaron por el regreso presencial hasta el ciclo escolar 2021-2022. Los estados de Chihuahua, Colima, Michoacán, Nayarit, Sinaloa y Zacatecas volverán a la modalidad presencial bajo esquema mixto al inicio del año 2022 (SEP, 2021).

En el boletín de la Secretaría de Educación Pública no. 146 (SEP, 2021), Delfina Gómez Álvarez afirmó que el calendario escolar será de 200 días de clase y el personal educativo tendrá 40 días de descanso. Para hacer frente al déficit escolar, la SEP ha ampliado el calendario escolar de 190 a 200 días lectivos, finalizando el año escolar el 28 de julio de 2022 (Párr. 2).

Se habla de cifras y de planes para la continuidad educativa y de los esfuerzos para transitar hacia las clases presenciales, sin embargo, todavía se desconocen los efectos secundarios de la pandemia. Es importante considerar los factores emocionales de una nueva realidad en las escuelas.

La Universidad Iberoamericana llevó a cabo una investigación a través del Instituto de Investigaciones para el Desarrollo con Equidad (EQUIDE), que presenta los primeros resultados de la Encuesta de Seguimiento de los Efectos del Covid-19 en el Bienestar de los Hogares Mexicanos (ENCOVID-19). En estos resultados se muestra que el 27.3\% de las personas de 18 años o más, presentaron síntomas de depresión, y el 32.4\%, síntomas graves de ansiedad. Los profesores deberán dar acompañamiento a jóvenes que quizás sufran ataques de ansiedad por la forma en que los afectó la pandemia, ya sea por la crisis económica, el encierro o por haber perdido a un familiar. Lo más probable es que los profesores no estén preparados para esta situación (Ortiz Cotte, 2021).

Por este motivo, es fundamental que el plan para el regreso a clases presenciales que diseñen las instituciones incluya un test diagnóstico de la salud emocional de alumnos y 
maestros; así habrá un antecedente para poder enfrentar los conflictos en el aula de la mejor manera. Las aulas virtuales, aún las mejores diseñadas, nunca podrán reemplazar la instrucción en el aula, particularmente en cursos donde la dinámica de grupo es fundamental para el proceso de aprendizaje (de Oliveira Dias et al., 2020).

En todos los estados de la República Mexicana se están llevando a cabo acciones para un regreso a la escuela de forma segura. Arvea Damián (2021) describe a detalle el Programa Pedagógico Emergente, conformado por protocolos y proyectos pedagógicos en situación de pandemia y confinamiento, desarrollado en Oaxaca. Este establece que estos protocolos aplican a la totalidad de la Zona Escolar y se conformó un Colectivo para integrarlos y supervisarlos. Los protocolos abarcan tanto procedimientos de higiene como acciones que se desarrollan en el salón de clases, el espacio escolar, el hogar y la comunidad. Los protocolos incluyen desde actividades académicas como reflexiones sobre el confinamiento a partir de lecturas, hasta actividades prácticas como la elaboración de cubrebocas, además de actividades comunitarias como el cultivo de huertos. El objetivo es que, a pesar de todos los inconvenientes de la pandemia, los estudiantes puedan desarrollar su inteligencia, comunicación y creatividad.

La UNICEF publicó los protocolos y lineamientos para la reapertura de guarderías, jardines infantiles, escuelas primarias y secundarias públicas y privadas en los países de América Latina y el Caribe (UNICEF, 2021b). De entre estos protocolos destaca el diseñado por el gobierno de las Islas Vírgenes, en donde se considera extender el cierre escolar en caso de brotes. También refuerza la práctica de higiene saludable, posponiendo reuniones y eventos no indispensables, e implementando múltiples estrategias de distanciamiento social, como evitar mezclar a los estudiantes en áreas comunes (HEOC, 2020).

La OMS, UNICEF y la UNESCO han aceptado un conjunto de ocho recomendaciones de expertos para la educación emitidas por el Grupo Asesor Técnico Europeo de la OMS para COVID-19 para ayudar a mantener las escuelas abiertas y seguras. Esas recomendaciones son las siguientes: 1) se espera que las escuelas estén entre las últimas en cerrar y las primeras en reabrir; 2) establecer una estrategia de prueba; 3) asegurarse de que existan procedimientos adecuados de mitigación de riesgos; 4) garantizar el bienestar mental y social; 5) proteger a los niños más vulnerables y marginados; 6) mejorar el ambiente de aprendizaje en la escuela; 7) involucrar a los niños y adolescentes 
en la toma de decisiones y, 8) implementar un programa de vacunación dirigido a mantener a los niños en la escuela (UNICEF, 2021a).

En este escenario, no solo los estudiantes han sido afectados. Los padres de familia en México consideran que no están listos para que sus hijos regresen a las clases presenciales, pues siguen temiendo al contagio.

Aunque reconocen que llevar a sus hijos a la escuela sería ser de mucha ayuda para los padres que trabajan fuera del hogar, ya que la institución educativa se convierte en un lugar seguro para su cuidado. Por otro lado, los padres de familia afirman que el exceso de tareas con las que los docenes llenaron los espacios vacíos ha resultado en más trabajo para ellos. La realidad actual es un tema complejo, con muchas variables por resolver, entre ellas la formación en temas pedagógicos de virtualidad, TI, programas académicos desarrollados para la presencialidad que choca con la impartición virtual (Martínez, 2020).

Para dar paso al regreso presencial a clases, es indispensable reconocer los diferentes aspectos y variables de este tema. La seguridad debe ser el objetivo número uno durante la transición de regreso a las clases. Los gobiernos e instituciones han apoyado y ayudado con el proceso de vacunación de estudiantes, docentes y personal administrativo, y las escuelas han promovido la concienciación sobre los síntomas, animando a cualquier persona sintomática a realizarse la prueba PCR. Universidades a nivel mundial cuentan con protocolos Covid-19 en sus páginas web para apoyar e informar a sus alumnos, exalumnos y docentes, no solo en aspectos de seguridad y concientización de síntomas, sino también en calendarios de vacunación. Algunos ejemplos son Oxford, UCLA (Universidad de California en Los Ángeles), Universidad Estatal de Oregón, Universidad de Nueva York, Universidad Estatal de Arizona, Universidad de Manitoba, Universidad de Georgetown, UNAM (Universidad Nacional Autónoma de México), entre muchas otras.

Una universidad privada mexicana, El Instituto Tecnológico y de Estudios Superiores de Monterrey, dio a sus estudiantes la opción de un Modelo HyFlex+Tec, que brindaba dos opciones: aprendizaje a distancia con clases en línea o aprendizaje híbrido con una combinación de aprendizaje a distancia y actividades presenciales. Independientemente de la opción que eligieran, los cursos comenzaron el 10 de agosto de 2021 y, al menos durante el primer mes, el cien por ciento de las clases se impartieron a distancia. El 
formato híbrido se irá implementando paulatinamente en otros campus cuando el nivel de alerta epidemiológica lo permita. Si no es posible estudiar en persona debido a la pandemia, el estudiante puede completar el semestre en su totalidad a través del aprendizaje remoto (Treviño, 2020).

Es necesario pensar en las futuras situaciones posibles. Los procesos para implementar las estrategias de regreso a clases presenciales después del confinamiento representan varios retos. Pérez-Archundia et al. (2020) hacen una reflexión sobre las consecuencias que podemos anticipar, las cuales tienen un impacto en el quehacer docente. Lo primero, es reconocer que la pandemia no se ha ido. No solo se deberán acatar las medidas sanitarias, sino que se presentarán retos didácticos, económicos y de relaciones humanas. Los autores consideran que tanto directivos como docentes deberán ser sensibles a las pérdidas de los seres queridos de los estudiantes, así como la pérdida de trabajo que conlleva dificultades económicas que puedan ocasionar que muchos de ellos deserten. También subrayan la necesidad de tener un plan de apoyo emocional para maestros y estudiantes que les permita llevar a buen término las actividades académicas, en donde la tecnología sea utilizada para el bien común.

Por otro lado, como lo apunta Casanova Cardiel (2020): "estamos ante una nueva generación de alumnos que, en general, está vinculada con la tecnología digital, lo cual ha modificado sus formas de aprender, sus intereses y sus habilidades. Sin embargo, esto no significa que puedan aprender con la tecnología; saben usarla para comunicarse, para interactuar en las redes sociales, pero no necesariamente la emplean como un recurso de aprendizaje" (p. 22). Esto se traduce en uno de los mayores retos en un escenario en el que los estudiantes encontraron en la tecnología su forma de aprender, pero no en todos los casos de forma efectiva. Que todos los estudiantes desarrollen todas las competencias necesarias de acuerdo con su nivel, será uno de los grandes objetivos del regreso a las aulas.

\section{CONCLUSIONES}

La planificación detallada para el regreso presencial a clases y las medidas preventivas para frenar los contagios de Covid-19 son necesarias para poder migrar hacia la educación presencial. Sin embargo, existe una tendencia importante a nivel internacional al retorno inmediato a clases presenciales o al sistema híbrido. Los diferentes contextos sociales son definitivos para el éxito de la implementación, especialmente en cuanto a las medidas de 
higiene y seguridad en la escuela, y la cuestión radica en que las instituciones deben garantizar estas condiciones. A pesar de los planes de retorno a clases, surge el factor fuera de toda previsión: los nuevos contagios, convirtiendo a la suspensión de las clases presenciales en una realidad en donde el sistema híbrido no avanza.

La forma para continuar las clases en todo el mundo dependerá del comportamiento de la pandemia, a pesar de las estrategias y protocolos implementados por gobiernos e instituciones. Más allá de todo esfuerzo, no podemos ignorar que el cuidado de los estudiantes y los maestros es más importante que un regreso presencial. Este es un tema que debe abrirse al diálogo para que, enfocándonos en lo que es vital, podamos tener una postura crítica, de apertura y de aprendizaje. La realidad cambió y con ella la necesidad de tener una actitud resiliente. Cuando hayamos superado la pandemia, volveremos a clases y recuperaremos el espacio de aprendizaje y de vida.

\section{LISTA DE REFERENCIAS}

Arvea Damián, M. (2021). Programa Pedagógico Emergente. Protocolos y Proyectos Pedagógicos en Situación de Pandemia y Confinamiento (Colectivo de la Zona 033. Nivel de Telesecundarias (ed.); 1st ed.). La Mano.

Cabrera Pérez, S. A. (2020). Reflexiones sobre la continuidad educativa ante situaciones de emergencia. In Universidad Iberoamericana Puebla (Ed.), Décimo Primer Coloquio Interinstitucional de Profesores (p. 7). https://repositorio.iberopuebla.mx/bitstream/handle/20.500.11777/4640/PIP_CA BRERA PEREZ_Susana Andrea_CC.pdf?sequence=1\&isAllowed=y

Casanova Cardiel, H. (2020). Educación y pandemia. Una visión académica (Universidad Nacional Autónoma de México (ed.); 1st ed.). Instituto de Investigaciones sobre la Universidad y la Educación.

Cervantes-González, E., \& Hernández López, M. S. (2020). El emprendimiento de padres de familia con la modalidad de clases en línea durante la pandemia del Covid-19. Emprenova, 1(2), 86-112.

Cucinotta, D., \& Vanelli, M. (2020). WHO declares COVID-19 a pandemic. Acta Biomedica, 91(1), 157-160. https://doi.org/10.23750/abm.v91i1.9397

De la Rosa, Y. (2020). Habrá clases presenciales incluso con semáforo rojo. Forbes, 1. https://www.forbes.com.mx/habra-clases-presenciales-incluso-con-semafororojo/ 
de Oliveira Dias, M., de Oliveira Albergarias Lopes, R., \& Correia Teles, A. (2020). Will Virtual Replace Classroom Teaching? Lessons from Virtual Classes via Zoom in the Times of COVID-19. Journal of Advances in Education and Philosophy, 04(05), 208-213. https://doi.org/10.36348/jaep.2020.v04i05.004

Farías Soares, L., \& Schoen, T. H. (2020). Medidas de prevenção à Covid-19 no retorno às aulas: protocolos de 13 países. Scielo Preprints. https://doi.org/10.1590/SciELOPreprints.1082

García-Peñalvo, F. J., \& Corell, A. (2020). La CoVId-19: ¿enzima de la transformación digital de la docencia o reflejo de una crisis metodológica y competencial en la educación superior? Campus Virtuales, 9(2), 83-98.

Goldstein, A. (2021). Glimpses of How Pandemic America Went Back to School. The New York Times. https://www.nytimes.com/2021/09/17/education/learning/schools-reopeningunited-states.html

González Fernández, K. (2021). Del aula presencial a la remota y de regreso: La enseñanza en situaciones de pandemia e incertidumbre. Revista Panamericana de Pedagogía, 31, 10-25. https://doi.org/10.21555/rpp.v0i31.2116

Guaman Chavez, R. E., Villareal Cobeña, Á. W., \& Cedeño Hidalgo, E. R. (2020). La Educación Híbrida como alternativa frente al Covid-19 en el Ecuador. TSE'DE Revista de Investigación Científica, 3(1), 134-147. http://tsachila.edu.ec/ojs/index.php/TSEDE/article/view/27/29

HEOC. (2020). School Reopening Guidance. https://bvi.gov.vg/sites/default/files/schools_reopening_guidance.pdf

Martínez, N. (2020, August 20). Ante Covid, padres no están preparados para el regreso a clases. $\quad$ El Sol de México. https://www.elsoldemexico.com.mx/mexico/sociedad/covid-19-coronaviruspandemia-padres-no-estan-preparados-regreso-clases-sep-nueva-normalidad5611582.html

Méndez Landa, F. J. (2021). La hibridación de clases sincrónicas y asincrónicas en la educación universitaria online: una estrategia para un mejor aprovechamiento del tiempo. In REDINE (Ed.), Medios digitales y metodologías docentes: Mejorar la educación desde un abordaje integral (pp. 74-82). Adaya Press. 
https://www.adayapress.com/wp-content/uploads/2021/06/medidoc8.pdf

Muñiz, C., \& Corduneanu, V. I. (2020). Percepción de riesgo y consumo mediático durante el inicio de la pandemia de Covid-19 en México. Más Poder Local, 41, 44-47. https://www.cdc.gov/mmwr/volumes/69/wr/pdfs/mm6924e1-H.pdf

Núñez, J. M., Reyes-Luna, A. M., Rodríguez-Fernández, A. de J., Jiménez-Ortega, A. D., Quiroz-Cázares, G., Balderas-Cruz, I., Lara-Pulido, J. A., Galeana-Pizaña, J. M., \& Sofia Seemann-Carús. (2021). Análisis territorial de las condiciones de la educación básica en México ante el regreso a clases presenciales en el marco del COVID-19. In Centrus y GeoLab (Vol. 1). https://ibero.mx/sites/default/files/educacion_basica.pdf

Ochoa Gutiérrez, R., \& Balderas Gutiérrez, K. (2021). Educación Continua en tiempos de pandemia, un reto para los maestros de inglés en universidades privadas en México. Revista Didasc@lia: D\&E., 12(3),152-164.

Ortiz Cotte, J. A. (2021). El regreso a clases. Síntesis. https://web.mediasolutions.mx/Notas/?id=202106241154142464\&temaid=1194 6

Pérez-Archundia, E., Rodríguez Marcial, R., \& Zarza Arizmendi, E. (2020). Voces y emociones de actores educativos ante la pandemia por la COVID-19 (Siglo XXI (ed.); 1st ed., Issue 1). EVG Editores.

Rubio Álvarez, A., Ballén Cifuentes, O. A., Blanco López, C. A., Bermúdez Guitiérrez, L. M., Cortés, R. A., Palacio Castañeda, J. A., \& Martínez Marulanda, C. E. (2020). El regreso a las aulas en el marco del Covid-19. In Nota Política Pública. No. 1. (Issue 1).

SEP. (2021). Boletín SEP no. 146 Mantiene SEP expectativa de iniciar el próximo ciclo escolar de manera presencial. https://www.gob.mx/sep/articulos/boletin-sep-no146-mantiene-sep-expectativa-de-iniciar-el-proximo-ciclo-escolar-de-manerapresencial? state $=$ published

Simon, D. (2020). In a Tokyo school, temperature checks and silent lunches as Japan restarts classes. The Washington Post. https://www.washingtonpost.com/world/asia_pacific/japan-coronavirus-schoolsreopen/2020/06/06/9047be8c-a645-11ea-8681-7d471bf20207_story.html

Taylor, A. (2020). As Trump pressures schools to reopen, here's how other countries have 
handled the return to the classroom. The Washington Post. https://www.washingtonpost.com/world/2020/06/05/coronavirus-countriesreopening-schools/

Treviño, R. (2020, July 22). Are you a Tec student? Here's the return to classes with HyFlex+Tec. Conecta News Desk. https://tec.mx/en/news/national/institution/are-you-tec-student-heres-returnclasses-hyflextec

Trilla, A. (2020). Un mundo, una salud: la epidemia por el nuevo coronavirus COVID19. Medicina Clinica, 154(5), 175-177. https://doi.org/10.1016/j.medcli.2020.02.002

U.S. Department of Education. (2021). Return to School Roadmap. A guide for K-12 schools and communities for the 2021-2022 school year. https://sites.ed.gov/roadmap/

Unesco. (2021). Education: From disruption to recovery. COVID-19. https://en.unesco.org/covid19/educationresponse

UNICEF. (2021a). Back to school. Information for children, parents and professionals. https://www.unicef.org/romania/what-does-return-school-during-covid-19pandemic-look

UNICEF. (2021b). Protocols and guidelines for the reopening of schools in Latin America and the Caribbean countries. https://www.unicef.org/lac/en/protocolsand-guidelines-reopening-schools-latin-america-and-caribbean-countries 